Note

\title{
Variance in neurocognitive performance is associated with dysbindin-1 in schizophrenia: A preliminary study
}

\author{
Gary Donohoe $^{a, b, *}$, Derek W. Morris ${ }^{b}$, Sarah Clarke ${ }^{b}$, Kevin A. McGhee ${ }^{b}$, Siobhan Schwaiger $^{b}$, \\ Jeanne-Marie Nangle ${ }^{\mathrm{b}}$, Hugh Garavan ${ }^{\mathrm{a}}$, Ian H Robertson ${ }^{\mathrm{a}}$, Michael Gill ${ }^{\mathrm{b}}$, Aiden Corvin ${ }^{\mathrm{b}, *}$ \\ ${ }^{a}$ Department of Psychology \& Trinity Institute of Neuroscience, Trinity College Dublin, Dublin, Ireland \\ ${ }^{\mathrm{b}}$ Neuropsychiatric Genetics Research Group, Department of Psychiatry and Institute of Molecular Medicine, \\ Trinity College Dublin, Dublin, Ireland \\ Received 30 January 2006; received in revised form 2 April 2006; accepted 1 June 2006 \\ Available online 22 August 2006
}

\begin{abstract}
Susceptibility genes for schizophrenia have been hypothesised to mediate liability for the disorder at least partly by influencing cognitive performance. We investigated the association between genotype and cognitive performance for a Dysbindin risk haplotype which is associated with schizophrenia in our sample. Fifty-two patients with schizophrenia or schizoaffective disorder (24 risk haplotypes carriers versus 28 non-risk haplotype carriers) were assessed in areas of cognition showing evidence of familial deficits in schizophrenia. Verbal and spatial memory, working memory, and attentional control was assessed using selected measures from the Weschler memory scale (WMS), Cambridge automated test battery (CANTAB), continuous performance test (CPT), and a simple go/no-go task. Pre-morbid IQ was also assessed using the Weschler Test of Adult Reading (WTAR). Patients carrying the Dysbindin risk haplotype showed significantly lower spatial working memory performance than patients who were non-risk carriers, with genotype explaining $12 \%$ of variance in performance. Our study suggests that the increased risk for schizophrenia associated with dysbindin may be partly mediated by its influence on pre-frontal function.
\end{abstract}

(c) 2006 Elsevier Ltd. All rights reserved.

Keywords: Dysbindin; Schizophrenia; Attention; Memory

\section{Introduction}

Deficits in cognitive performance are increasingly viewed as core to schizophrenia and highly predictive of functional outcome (Green, 1996; Heinrichs, 2005). The heritability of performance deficits on measures of executive function, vigilance, and declarative memory has been demonstrated in twin studies (Cannon et al., 2000; Goldberg et al., 1990) and in studies of first degree relatives (Egan et al., 2001; Faraone et al., 1995). This has lead to the suggestion that genetic liability for schizophrenia is at least partly mediated by deleterious effects on the brain regions associated with these cognitive functions, including pre-frontal and medial temporal lobe regions (Weinberger et al., 2001).

\footnotetext{
* Correspondence to: Neuropsychiatric Genetics Research Group, Department of Psychiatry, Trinty Health Science Building, St. James' Hospital, Dublin 8, Ireland. Tel.: +353 1608 3272; fax: +3531608 3405 .

E-mail addresses: donoghug@tcd.ie (G. Donohoe), acorvin@tcd.ie (A. Corvin).
}

Recent reviews of schizophrenia genetics (Craddock, O'Donovan, \& Owen, 2005) have highlighted the potential role of a number of candidate genes in conferring liability to the disorder. Among these, an association with dystrobrevin-binding protein 1 (dysbindin), a $40-50-\mathrm{kDa}$ protein that is expressed in neurons throughout mouse and human brain, has been one of the most widely replicated findings, including in our own casecontrol sample (e.g. Schwab et al., 2003; Straub et al., 2002; Williams et al., 2004).

Although the biological significance of dysbindin remains unclear, a number of lines of evidence suggest that it may have a role in cognition. Firstly, using a cognitive phenotype, two studies have identified linkage to loci on chromosome $6 \mathrm{p}$ that are, respectively, overlapping with and adjacent to the dysbindin gene (Hallmayer et al., 2005; Posthuma et al., 2005). Secondly, two recent post-mortem studies have reported specific reductions in the expression of dysbindin in the hippocampal region (Talbot et al., 2004) and the dorsolateral pre-frontal cortex (Weickert et al., 2004) of deceased patients with schizophre- 
nia. Talbot et al. (2004) and Numakawa et al. (2004) reported evidence that dysbindin is impacting on glutamate neurotransmission, which is associated with a number of cortical processes underpinning neurocognition, including hippocampal long term potentiation (Poo, 2001; Tyler, Alonso, Bramham, \& PozzoMiller, 2002). Thirdly, dysbindin has recently been associated with variance in cognitive function in schizophrenia based on an index of general cognitive ability (Burdick et al., 2006).

We investigated the potential relationship between dysbindin haplotype carrier status and selected measures of neurocognitive impairments showing evidence of familiality in schizophrenia, namely episodic memory, working memory, and attentional control. We expected that if the risk for schizophrenia associated with dysbindin is at least partly mediated by its influence on neurocognitive function, then carriers of the risk haplotype will perform significantly worse on at least some neurocognitive measures.

\section{Method}

\subsection{Subjects}

After approval was obtained from the relevant hospital ethics committees, we re-contacted patients from our original genetic sample $(n=359)$ who were still attending community psychiatric services in their local catchment area and who met our inclusion criteria (below). This sample was comprised entirely of patients of Irish origin (having Irish parents/grandparents). DSM-IV diagnosis was confirmed by a psychiatrist or psychiatric nurse trained to use the Structured Clinical Interview for DSM (SCID; American Psychiatric Association, 1992).

Eligibility for neurocognitive assessment required patients to satisfy (based on interview and chart review) the following additional criteria: (a) aged between 18 and 60 years, (b) no history of substance abuse in the preceding 6 months, (c) no other psychiatric diagnosis, (d) no history of head injury with loss of consciousness, and (e) no history of epilepsy or seizures. In addition, patients were only approached if they were outpatients and, in the view of their treatment team, clinically stable. Based on these criteria, neurocognitive data was ascertained on 91 of our total schizophrenia genetic sample. All cases gave written informed consent prior to undergoing neurocognitive assessment.

\subsection{Neurocognitive measures}

Verbal episodic and working memory was assessed using the Logical Memory and Letter Number Sequencing sub-tests from the Weschler Memory Scales, 3rd edition (WMS-III; Weschler, 1998). Spatial memory recall and working memory was assessed using the Paired Associate Learning task (PAL) and the Spatial Working Memory task (SWM) from the Cambridge Automated Test Battery (CANTAB; Cambridge Cognition, 2003). Attentional control was assessed using the distractibility version of Gordon's Continuous Performance Test (CPT; Gordon, 1996), and the CANTAB Intra-Extra Dimensional Set Shifting Task (IED). We also measured ability to inhibit response to a pre-potent response using a simple Go/No-Go task (Garavan, Ross, \& Stein, 1999). In this task participants attended to a series of alternating stimuli (' $\mathrm{X}$ ' or ' $\mathrm{Y}$ ') with the requirement to press a button for each stimuli except where the same stimulus was presented twice (' $X$ ' followed by ' $X$ ' or ' $Y$ ' followed by ' $Y$ '). To enable use of pre-morbid IQ as a co-variable, the Weschler test of Adult Reading (WTAR; 2001) was also administered.

\subsection{Genotyping}

The dysbindin 'risk' haplotype identified by Williams et al. (2004) is derived from alleles $C-A-T$ at SNPs P1655 (rs2619539), P1635 (rs3213207) and SNP66961 (rs2619538). All SNPs were genotyped using Custom Taqman ${ }^{\circledR}$ SNP Genotyping Assays from Applied Biosystems on an ABI Prism 7900HT
Sequence Detection System. In order to compare dysbindin at-risk haplotype carriers (estimated frequency of 0.21 in cases in the overall sample) with noncarriers, assignment of haplotype carrier status to individual cases was performed using the Bayesian approach to reconstructing haplotypes implemented in the programme PHASE (v2.0.2; Stephens \& Donnelly, 2003). A case was only included in the study where the identity of its two haplotypes could be determined at $p>0.95$. On this basis we successfully identified 24 risk haplotype carrier and 28 non-risk carriers.

\subsection{Statistical analysis}

Dysbindin risk haplotype carriers were compared with non-carriers on each neurocognitive measure using one-way ANCOVAs and controlling for variables associated with neurocognitive performance in our sample (age and pre-morbid IQ). Significant associations with genotype were further analysed in a regression analysis to determine the variance in cognitive performance associated with genotype.

A critical issue in assessing the significance of genetic association with phenotypic measures is the likelihood of type I errors. Because of the presumably small effects of specific genes in complex genetic disorders, and that fact that performance on at least some cognitive tests will be correlated, we did not use a Bonferroni correction because of its overly stringent nature. Instead we sought to minimise the possibility of type I error by: (a) only investigating the risk haplotype already associated with schizophrenia in our sample, and (b) setting the $\alpha$-value for statistical significance at 0.01 .

\section{Results}

Mean scores on demographic and neurocognitive variables are presented for both risk haplotype and non-haplotype groups in Table 1. Demographic characteristics are described separately for each genotype group. While the two groups did not differ on the demographic and clinical variables presented, predictably, many cognitive variables correlated significantly with age and pre-morbid IQ. Age was significantly negatively correlated with verbal and spatial working memory, and CPT performance. Premorbid IQ was positively correlated with verbal and spatial memory recall, and with verbal and spatial working memory. On this basis both age and pre-morbid IQ were entered as covariables in analysis of between group variance in cognitive performance.

To test the hypothesis that the Dysbindin risk haplotype would be associated with poorer performance on cognitive measures we undertook a series of one-way ANCOVAs where Dysbindin risk haplotype carrier status was the grouping variable. In each analysis cognitive task performance was taken as the dependent measure, and age and pre-morbid IQ entered as co-variables (see Table 1).

On this basis, Dysbindin risk haplotype carriers showed significantly poorer performance in spatial working memory (as measured by the CANTAB SWM task). This was in the absence of a similar association between risk haplotype carrier status and verbal working memory as measured by the Letter Number Sequencing task.

A trend for risk haplotype carriers to perform better on a simple go/no-go measure of response inhibition was also observed (see Fig. 1). No differences between risk and non-risk haplotype carrier groups were observed for either measure of attentional control (CPT or IDED tasks), or for either verbal or spatial memory recall (Logical Memory sub-test or PAL tasks). 
Table 1

Differences in mean score between carriers of the Dysbindin risk haplotype carrier vs. non-carriers

\begin{tabular}{|c|c|c|c|c|}
\hline Dysbindin & $\begin{array}{l}\text { Risk haplotype }(n=24) \text {, } \\
\text { mean (S.D.) }\end{array}$ & $\begin{array}{l}\text { Non-risk haplotype }(n=28) \text {, } \\
\text { mean (S.D.) }\end{array}$ & $t$ or $\chi^{2}$ & $p$ \\
\hline Age & $44.9(9.9)$ & $44.4(10.1)$ & 0.175 & 0.862 \\
\hline Male (\%) & $17(44.7)$ & $21(55.3)$ & 0.008 & 0.994 \\
\hline Pre-morbid IQ & $93.0(12.3)$ & $93.1(11.4)$ & 0.056 & 0.956 \\
\hline Years in education & $13.3(3.3)$ & $12.7(2.3)$ & 0.702 & 0.486 \\
\hline Age at onset & $21.1(5.2)$ & $22.8(5.5)$ & 1.05 & 0.299 \\
\hline Aty. neuroleptic (\%) & $12(60)$ & $14(52)$ & 0.579 & 0.767 \\
\hline Anti-cholinergic (\%) & $3(15)$ & $2(7.4)$ & 0.404 & 0.638 \\
\hline Memory functioning & & & $\boldsymbol{F}$ & \\
\hline Logical memory I & $27.67(12.1)$ & $26.67(12.0)$ & 0.012 & 0.912 \\
\hline Logical memory II & $13.62(7.0)$ & $14.87(10.2)$ & 1.00 & 0.123 \\
\hline PAL stages & $6.7(1.8)$ & $7.26(1.0)$ & 2.09 & 0.115 \\
\hline PAL Adj. errors & $79.83(67.3)$ & $59.89(46.7)$ & 1.75 & 0.112 \\
\hline \multicolumn{5}{|l|}{ Attention } \\
\hline CPT (distractors) accuracy & $18.05(8.9)$ & $16.67(9.0)$ & 0.023 & 0.880 \\
\hline ID/ED stage 6 errors & $0.81(1.28)$ & $0.46(0.51)$ & 1.27 & 0.267 \\
\hline ID/ED stage 8 errors & $20.75(6.95)$ & $21.17(7.88)$ & 0.149 & 0.701 \\
\hline Go/no-go accuracy & $22.38(6.8)$ & $16.83(7.7)$ & 4.377 & 0.044 \\
\hline \multicolumn{5}{|l|}{ Working memory } \\
\hline LN sequencing & $7.39(3.6)$ & $7.96(3.4)$ & 1.153 & 0.289 \\
\hline SWM errors & $64.74(19.0)$ & $49.88(22.0)$ & 7.394 & 0.009 \\
\hline
\end{tabular}

Bold values signify that the results obtained are statistically significant at $p=0.05$ and $p=0.01$.

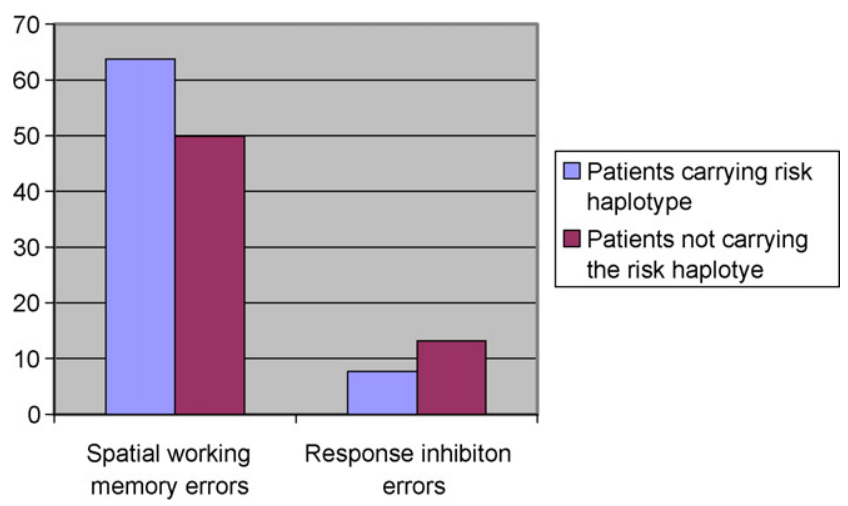

Fig. 1. Total number of (a) spatial working memory errors on the Cantab SWM task, and (b) response inhibition errors on the go/no-go task.

To estimate the variance in spatial working memory accounted for by dysbindin, we undertook a regression analysis. CANTAB spatial working memory error was taken as the dependent variable, age and pre-morbid IQ were entered on the first step prior to entering genotype. On this basis, dysbindin was estimated to explain $12 \%$ of variance in spatial working memory after the effects of age and IQ were partialled out $(F$ change $=7.39$; d.f. $=1.51 ; p=.009$ ).

\section{Discussion}

Carriers of the Dysbindin risk haplotype performed more poorly than non-carriers on the CANTAB spatial working memory task. An association with spatial working memory is interesting for a number of reasons. Working memory has consistently been shown to be impaired in schizophrenia and to be at least partly under genetic influence, on the basis of both family studies (Glahn et al., 2003; Myles-Worsley \& Park, 2002; Park, Holzman, \& Goldman-Rakic, 1995) and molecular studies (e.g. the association with the COMT VAL/MET polymorphism; Bilder et al., 2002; Goldberg et al., 2003). Poor performance on working memory tasks in schizophrenia have been associated with reduced activation in dorsolateral pre-frontal cortex (Goldman-Rakic, 1994; Weinberger et al., 2001). Given the existing evidence from the post-mortem study by Weickert et al. (2004) that dysbindin is under-expressed in dorsolateral prefrontal cortex, an influence on this cortical region may be one mechanism by which dysbindin is influencing cognitive function, and possibly also increasing liability for schizophrenia.

The neurophysiological basis of WM has also been widely investigated, and the importance of dopamine in regulating WM consistently reported (Castner, Goldman-Rakic, \& Williams, 2004; Goldman-Rakic, 1999). Neuropharmacological models are increasingly moving towards an integrated view of schizophrenia as a disorder of regulation and interaction of dopaminergic and glutamatergic systems (Moghaddam \& Krystal, 2003). Given the evidence that dysbindin is involved in glutamate function (Numakawa et al., 2004; Talbot et al., 2004), it is interesting to speculate about whether dysbindin may be influencing dopamine function in pre-frontal cortex.

Given the association between dysbindin and an index of more general cognitive ability recently reported (Burdick et al., 2006), our finding of association with spatial working memory raises an important question regarding the specificity of dysbindin's role in cognition. We have previously shown that variance in working memory performance is associated with general cognitive performance in schizophrenia, unlike attentional control (Donohoe et al., 2006a). Given that Burdick et al. 
(2006) sought to index general cognitive ability rather than specific processes such as spatial working memory, the difference in association may simply reflect the difference in approach to cognitive task measurement. It is also interesting to note that our finding with working memory was specific to spatial working memory. Further study will be helpful in determining whether dysbindin's influence on cognition is specific to visuo-spatial information systems only (implicating right pre-frontal cortex), or whether verbal processes are also involved.

In this exploratory investigation of dysbindin's role in cognition, we did not find an association between genotype and measures of attentional control or episodic memory. We did see a trend towards association between dysbindin and better go/nogo performance. Interestingly, Fallgatter et al. (2006), using a neurophysiological index of the same paradigm in a normal sample, also found evidence of association with two dysbindin SNP's previously associated with schizophrenia (Schwab et al., 2003; van den Oord et al., 2003). Investigation in a larger schizophrenia sample will be required to interpret the significance of these results.

Working memory performance has recently been associated with functional outcome in a number of schizophrenia studies (Greenwood, Landau, \& Wykes, 2005; Hofer et al., 2005; Revheim et al., 2006). This association has been strongest for patients with a preponderance of negative symptoms, and indeed negative symptoms have been found to correlate with performance deficits on measures of a variety of higher cognitive functions, including working memory (Donohoe et al., 2006a; Donohoe, Corvin, \& Robertson, 2006; Donohoe \& Robertson, 2003). It is therefore very interesting to note that the other main phenotype with which dysbindin has been associated is negative symptom severity (Derosse et al., 2006; Fanous et al., 2005). One interpretation of these data is that the Dysbindin risk haplotype may be contributing to the delineation of a subtype of schizophrenia characterised by a deficit syndrome (see also Gornick et al.'s (2005) study on dysbindin and poor pre-morbid adjustment).

This paper adds to the growing body of research on the genetics of memory and attention. Working memory has already been associated with variants in a number of genes, including COMT, DISC-1 and DAT1 (Callicott et al., 2005; Rybakowski et al., 2006), reflecting the likelihood that multiple genes are influencing working memory ability (Goldberg \& Weinberger, 2004). Our study provides further evidence that dysbindin may mediate risk for schizophrenia at least partly through a deleterious effect on working memory. We expect that further cognitive phenotype findings on this leading susceptibility gene for schizophrenia will continue to elucidate its potential role in the pathogenesis of the disorder.

\section{Acknowledgement}

Our sincere thanks to patients and hospital staff who collaborated in this study. This work was generously supported by research grants from Science Foundation Ireland, The Higher Educational Authority (HEA) Ireland, and the Health Research Board Ireland.

\section{References}

American Psychiatric Association. (1992). Structured clincial assessment for the DSM-III-R. Washington DC: American Psychiatric Association.

Bilder, R. M., Volavka, J., Czobor, P., Malhotra, A. K., Kennedy, J. L., Ni, X., et al. (2002). Neurocognitive correlates of the COMT Val(158)Met polymorphism in chronic schizophrenia. Biological Psychiatry, 52, 701-707.

Burdick, K. E., Lencz, T., Funke, B., Finn, C. T., Szeszko, P. R., Kane, J. M., et al. (2006). Genetic variation in DTNBP1 influences general cognitive ability. Human Molecular Genetics.

Callicott, J. H., Straub, R. E., Pezawas, L., Egan, M. F., Mattay, V. S., Hariri, A. R., et al. (2005). Variation in DISC1 affects hippocampal structure and function and increases risk for schizophrenia. Proceedings of the National Academy of Sciences of the United States of America, 102, 8627-8632.

Cambridge Cognition. (2003). Cambridge neuropsychological test automated battery, expedio version (CANTABexpedio). Cambridge: Cambridge cognition Ltd.

Cannon, T. D., Rosso, I. M., Hollister, J. M., Bearden, C. E., Sanchez, L. E., \& Hadley, T. (2000). A prospective cohort study of genetic and perinatal influences in the etiology of schizophrenia. Schizophrenia Bulletin, 26, 351-366.

Castner, S. A., Goldman-Rakic, P. S., \& Williams, G. V. (2004). Animal models of working memory: Insights for targeting cognitive dysfunction in schizophrenia. Psychopharmacology (Berlin), 174, 111-125.

Craddock, N., O’Donovan, M. C., \& Owen, M. J. (2005). The genetics of schizophrenia and bipolar disorder: Dissecting psychosis. Journal of Medical Genetics, 42, 193-204.

Derosse, P., Funke, B., Burdick, K. E., Lencz, T., Ekholm, J. M., Kane, J. M., et al. (2006). Dysbindin genotype and negative symptoms in schizophrenia. The American Journal of Psychiatry, 163, 532-534.

Donohoe, G., Clarke, S., Morris, D., Nangle, J. M., Schwaiger, S., Gill, M., et al. (2006). Are deficits in executive sub-processes simply reflecting more general cognitive decline in schizophrenia? Schizophr Res, 85(1-3), 168-173.

Donohoe, G., Corvin, A., \& Robertson, I. H. (2006). Evidence that specific executive functions predict symptom variance among schizophrenia patients with a predominantly negative symptom profile. Cognitive Neuropsychiatry, $11,13-32$.

Donohoe, G., \& Robertson, I. H. (2003). Can specific deficits in executive functioning explain the negative symptoms of schizophrenia? A review. Neurocase, 9, 97-108.

Egan, M. F., Goldberg, T. E., Gscheidle, T., Weirich, M., Rawlings, R., Hyde, T. M., et al. (2001). Relative risk for cognitive impairments in siblings of patients with schizophrenia. Biological Psychiatry, 50, 98-107.

Fallgatter, A. J., Herrmann, M. J., Hohoff, C., Ehlis, A. C., Jarczok, T. A., Freitag, C. M., et al. (2006). DTNBP1 (dysbindin) gene variants modulate prefrontal brain function in healthy individuals. Neuropsychopharmacology.

Fanous, A. H., van den Oord, E. J., Riley, B. P., Aggen, S. H., Neale, M. C., O'Neill, F. A., et al. (2005). Relationship between a high-risk haplotype in the DTNBP1 (dysbindin) gene and clinical features of schizophrenia. The American Journal of Psychiatry, 162, 1824-1832.

Faraone, S. V., Seidman, L. J., Kremen, W. S., Pepple, J. R., Lyons, M. J., \& Tsuang, M. T. (1995). Neuropsychological functioning among the nonpsychotic relatives of schizophrenic patients: A diagnostic efficiency analysis. Journal of Abnormal Psychology, 104, 286-304.

Garavan, H., Ross, T. J., \& Stein, E. A. (1999). Right hemispheric dominance of inhibitory control: An event-related functional MRI study. Proceedings of the National Academy of Sciences of the United States of America, 96, 8301-8306.

Glahn, D. C., Therman, S., Manninen, M., Huttunen, M., Kaprio, J., Lonnqvist, J., et al. (2003). Spatial working memory as an endophenotype for schizophrenia. Biological Psychiatry, 53, 624-626.

Goldberg, T.E., Egan, M. F., Gscheidle, T., Coppola, R., Weickert, T., Kolachana, B. S., et al. (2003). Executive subprocesses in working memory: Relationship to catechol-O-methyltransferase Val158Met genotype and schizophrenia. Archives of General Psychiatry, 60, 889-896.

Goldberg, T. E., Ragland, J. D., Torrey, E. F., Gold, J. M., Bigelow, L. B., \& Weinberger, D. R. (1990). Neuropsychological assessment of monozygotic twins discordant for schizophrenia. Archives of General Psychiatry, 47, 1066-1072. 
Goldberg, T. E., \& Weinberger, D. R. (2004). Genes and the parsing of cognitive processes. Trends Cogn Sci, 8(7), 325-335.

Goldman-Rakic, P. S. (1994). Working memory dysfunction in schizophrenia. The Journal of Neuropsychiatry and Clinical Neurosciences, 6, 348-357.

Goldman-Rakic, P. S. (1999). The physiological approach: functional architecture of working memory and disordered cognition in schizophrenia. Biol Psychiatry, 46(5), 650-661.

Gordon, M. (1996). Gordon Diagnostic System III. In Gordon diagnostic system. DeWitt, NY: Gordon Diagnostic Systems.

Gornick, M. C., Addington, A. M., Sporn, A., Gogtay, N., Greenstein, D., Lenane, M., et al. (2005). Dysbindin (DTNBP1, 6p22. 3) is associated with childhood-onset psychosis and endophenotypes measured by the Premorbid Adjustment Scale (PAS). Journal of Autism and Developmental Disorders, $35,831-838$.

Green, M. F. (1996). What are the functional consequences of neurocognitive deficits in schizophrenia? The American Journal of Psychiatry, 153, 321-330.

Greenwood, K. E., Landau, S., \& Wykes, T. (2005). Negative symptoms and specific cognitive impairments as combined targets for improved functional outcome within cognitive remediation therapy. Schizophrenia Bulletin, 31, 910-921.

Hallmayer, J. F., Kalaydjieva, L., Badcock, J., Dragovic, M., Howell, S., Michie, P. T., et al. (2005). Genetic evidence for a distinct subtype of schizophrenia characterized by pervasive cognitive deficit. American Journal of Human Genetics, 77, 468-476.

Heinrichs, R. W. (2005). The primacy of cognition in schizophrenia. The American Psychologist, 60, 229-242.

Hofer, A., Baumgartner, S., Bodner, T., Edlinger, M., Hummer, M., Kemmler, G., et al. (2005). Patient outcomes in schizophrenia II: The impact of cognition. European Psychiatry, 20, 395-402.

Moghaddam, B., \& Krystal, J. H. (2003). The neurochemistry of schizophrenia. In S. Hirsch, \& D. Weinberger (Eds.), Schizophrenia. Oxford, UK: Blackwell Publishing.

Myles-Worsley, M., \& Park, S. (2002). Spatial working memory deficits in schizophrenia patients and their first degree relatives from Palau, Micronesia. American Journal of Medical Genetics, 114, 609-615.

Numakawa, T., Yagasaki, Y., Ishimoto, T., Okada, T., Suzuki, T., Iwata, N., et al. (2004). Evidence of novel neuronal functions of dysbindin, a susceptibility gene for schizophrenia. Human Molecular Genetics, 13, 2699-2708.

Park, S., Holzman, P. S., \& Goldman-Rakic, P. S. (1995). Spatial working memory deficits in the relatives of schizophrenic patients. Archives of General Psychiatry, 52, 821-828.

Poo, M. M. (2001). Neurotrophins as synaptic modulators. Nature Reviews. Neuroscience, 2, 24-32.

Posthuma, D., Luciano, M., Geus, E. J., Wright, M. J., Slagboom, P. E., Montgomery, G. W., et al. (2005). A genomewide scan for intelligence identifies quantitative trait loci on 2q and 6p. American Journal of Human Genetics, 77, 318-326.

Revheim, N., Schechter, I., Kim, D., Silipo, G., Allingham, B., Butler, P., et al. (2006). Neurocognitive and symptom correlates of daily problem-solving skills in schizophrenia. Schizophrenia Research.

Rybakowski, J. K., Borkowska, A., Czerski, P. M., Dmitrzak-Weglarz, M., Skibinska, M., Kapelski, P., et al. (2006). Performance on the Wisconsin Card Sorting Test in schizophrenia and genes of dopaminergic inactivation (COMT, DAT NET). Psychiatry Res, 143(1), 13-19.

Schwab, S. G., Knapp, M., Mondabon, S., Hallmayer, J., Borrmann-Hassenbach, M., Albus, M., et al. (2003). Support for association of schizophrenia with genetic variation in the 6 p22.3 gene, dysbindin, in sib-pair families with linkage and in an additional sample of triad families. American Journal of Human Genetics, 72, 185-190.

Stephens, M., \& Donnelly, P. (2003). A comparison of bayesian methods for haplotype reconstruction from population genotype data. American Journal of Human Genetics, 73(5), 1162-1169.

Straub, R. E., Jiang, Y., MacLean, C. J., Ma, Y., Webb, B. T., Myakishev, M. V., et al. (2002). Genetic variation in the 6p22.3 gene DTNBP1, the human ortholog of the mouse dysbindin gene, is associated with schizophrenia. American Journal of Human Genetics, 71, 337-348.

Talbot, K., Eidem, W. L., Tinsley, C. L., Benson, M. A., Thompson, E. W., Smith, R. J., et al. (2004). Dysbindin is reduced in intrinsic, glutamatergic terminals of the hippocampal formation in schizophrenia. The Journal of Clinical Investigation, 113, 1353-1363.

Tyler, W. J., Alonso, M., Bramham, C. R., \& Pozzo-Miller, L. D. (2002). From acquisition to consolidation: On the role of brain-derived neurotrophic factor signaling in hippocampal-dependent learning. Learning and Memory, 9 , 224-237.

van den Oord, E. J., Sullivan, P. F., Jiang, Y., Walsh, D., O’Neill, F. A., Kendler, K. S., et al. (2003). Identification of a high-risk haplotype for the dystrobrevin binding protein 1 (DTNBP1) gene in the Irish study of high-density schizophrenia families. Molecular Psychiatry, 8, 499-510.

Weickert, C. S., Straub, R. E., McClintock, B. W., Matsumoto, M., Hashimoto, R., Hyde, T. M., et al. (2004). Human dysbindin (DTNBP1) gene expression in normal brain and in schizophrenic prefrontal cortex and midbrain. Archives of General Psychiatry, 61, 544-555.

Weinberger, D. R., Egan, M. F., Bertolino, A., Callicott, J. H., Mattay, V. S., Lipska, B. K., et al. (2001). Prefrontal neurons and the genetics of schizophrenia. Biological Psychiatry, 50, 825-844.

Weschler, D. (1998). Weschler memory scale (3rd ed. (WAIS-III)). New York: Psychological Corporation.

Williams, N. M., Preece, A., Morris, D. W., Spurlock, G., Bray, N. J., Stephens, M., et al. (2004). Identification in 2 independent samples of a novel schizophrenia risk haplotype of the dystrobrevin binding protein gene (DTNBP1). Archives of General Psychiatry, 61, 336-344. 